\title{
Spin polarization and g-factor enhancement in graphene nanoribbons in a magnetic field
}

\author{
S Ihnatsenka and Igor Zozoulenko
}

\section{Linköping University Post Print}

N.B.: When citing this work, cite the original article.

Original Publication:

S Ihnatsenka and Igor Zozoulenko, Spin polarization and g-factor enhancement in graphene nanoribbons in a magnetic field, 2012, Physical Review B. Condensed Matter and Materials Physics, (86), 15, 155407.

http://dx.doi.org/10.1103/PhysRevB.86.155407

Copyright: American Physical Society

http://www.aps.org/

Postprint available at: Linköping University Electronic Press

http://urn.kb.se/resolve?urn=urn:nbn:se:liu:diva-85196 


\title{
Spin polarization and $g$-factor enhancement in graphene nanoribbons in a magnetic field
}

\author{
S. Ihnatsenka* \\ Department of Physics, Simon Fraser University, Burnaby, British Columbia, Canada V5A IS6 \\ I. V. Zozoulenko ${ }^{\dagger}$ \\ Solid State Electronics, ITN, Linköping University, 601 74, Norrköping, Sweden
}

(Received 27 June 2012; published 5 October 2012)

\begin{abstract}
We provide a systematic quantitative description of spin polarization in armchair and zigzag graphene nanoribbons (GNRs) in a perpendicular magnetic field. We first address spinless electrons within the Hartree approximation, studying the evolution of the magnetoband structure and formation of the compressible strips. We discuss the potential profile and the density distribution near the edges and the difference and similarities between armchair and zigzag edges. Accounting for the Zeeman interaction and describing the spin effects via the Hubbard term, we study the spin-resolved subband structure and relate the spin polarization of the system at hand to the formation of the compressible strips for the case of spinless electrons. At high magnetic field the calculated effective $g$ factor varies around a value of $\left\langle g^{*}\right\rangle \approx 2.25$ for armchair GNRs and $\left\langle g^{*}\right\rangle \approx 3$ for zigzag GNRs. An important finding is that in zigzag GNRs the zero-energy mode remains pinned to the Fermi energy and becomes fully spin polarized for all magnetic fields, which, in turn, leads to a strong spin polarization of the electron density near the zigzag edge. Because of this the effective $g$ factor in zigzag GNRs is strongly enhanced at low fields reaching values up to $g^{*} \approx 30$. This is in contrast to armchair GNRs, where the effective $g$ factor at low field is close to its bare value, $g=2$.
\end{abstract}

DOI: 10.1103/PhysRevB.86.155407

PACS number(s): 72.80.Vp, 73.22.Pr, 73.63.Nm, 73.43.-f

\section{INTRODUCTION}

Investigation of the effects of electron interaction and spin in graphene at high magnetic field represents one of the frontiers in graphene research. Even though many aspects of the magnetoconductance of graphene related to the formation of unconventional Landau level spectra and the anomalous Hall effect are well understood theoretically and confirmed experimentally, ${ }^{1-4}$ there are still a number of questions awaiting resolution. One of these questions which is extensively debated in the current literature is the origin of the splitting of the lowest Landau level and the emergence of the insulating state at the Dirac point. ${ }^{5-9}$ Even though the precise origin of this state is under current debate, it is generally believed that it is related to electron-electron interaction and spin effects. The importance of electron interaction was also outlined for higher Landau levels. ${ }^{6}$ Recently, spin splitting in graphene and bilayer graphene in high magnetic field was experimentally analyzed by Kurganova et al., ${ }^{10}$ who found that the $g$ factor in graphene is enhanced and attributed this to electron-electron interaction effects. The spin splitting of the states in graphene ${ }^{11}$ and graphene quantum dots ${ }^{12}$ was also studied in a parallel magnetic field.

Motivated by this interest in the electron interaction and spin effects in graphene in the high magnetic field, in the present paper we study the spin polarization and enhancement of the $g$ factor in graphene nanoribbons (GNRs). Note that various aspects of electron and spin interactions in the high magnetic field have been extensively studied in conventional semiconducting quantum wires defined in a two-dimensional electron gas (2DEG). ${ }^{13-21}$ One of the motivations for such studies is related to advances in semiconductor spintronics utilizing the spin degree of freedom for adding new functionalities to electronic devices. ${ }^{22}$ Some proposed and investigated devices for spintronics and quantum computation applications operate in the edge-state regime, ${ }^{23,24}$ which obviously requires a detailed knowledge of the structure of the states in a quantum wire or at the edge of the 2DEG. The properties of and detailed information about propagating states at the boundaries are also essential for interpretation of experiments in various electron interferometers in the quantum Hall regime. ${ }^{25-27}$ Because graphene represents a very promising system for implementation of many devices and concepts for spintronics and quantum information processing applications utilizing the edge-state transport regime, a detailed knowledge of the density and potential profiles near the edges as well as spin properties is important for understanding and designing such devices.

This paper is organized as follows. In Sec. II we present a formulation of the problem, define the Hamiltonian, and briefly outline the self-consistent computational scheme. The results and discussion are presented in Sec. II. Section II A discusses the potential profile and the charge accumulation near the edges in ribbons of different widths and edge terminations. Section II B is devoted to the case of spinless electrons, focusing on the formation of compressible strips and the evolution of the magnetoband structure. Finally, based on the results of Sec. II B, Sec. II C discusses the spin splitting and the enhancement of the $g$ factor for the case of electrons with spin. The conclusions of this work are presented in Sec. III.

\section{MODEL}

We consider an infinite GNR of width $W$, located in an insulating substrate with relative permittivity $\epsilon_{r}$ and subjected to perpendicular magnetic field $B$ [see inset in Fig. 1(b)]. A metallic back gate situated at a distance $d$ from the ribbon is used to tune the Fermi energy in order to change an electron concentration in the GNR. The system is modeled by 

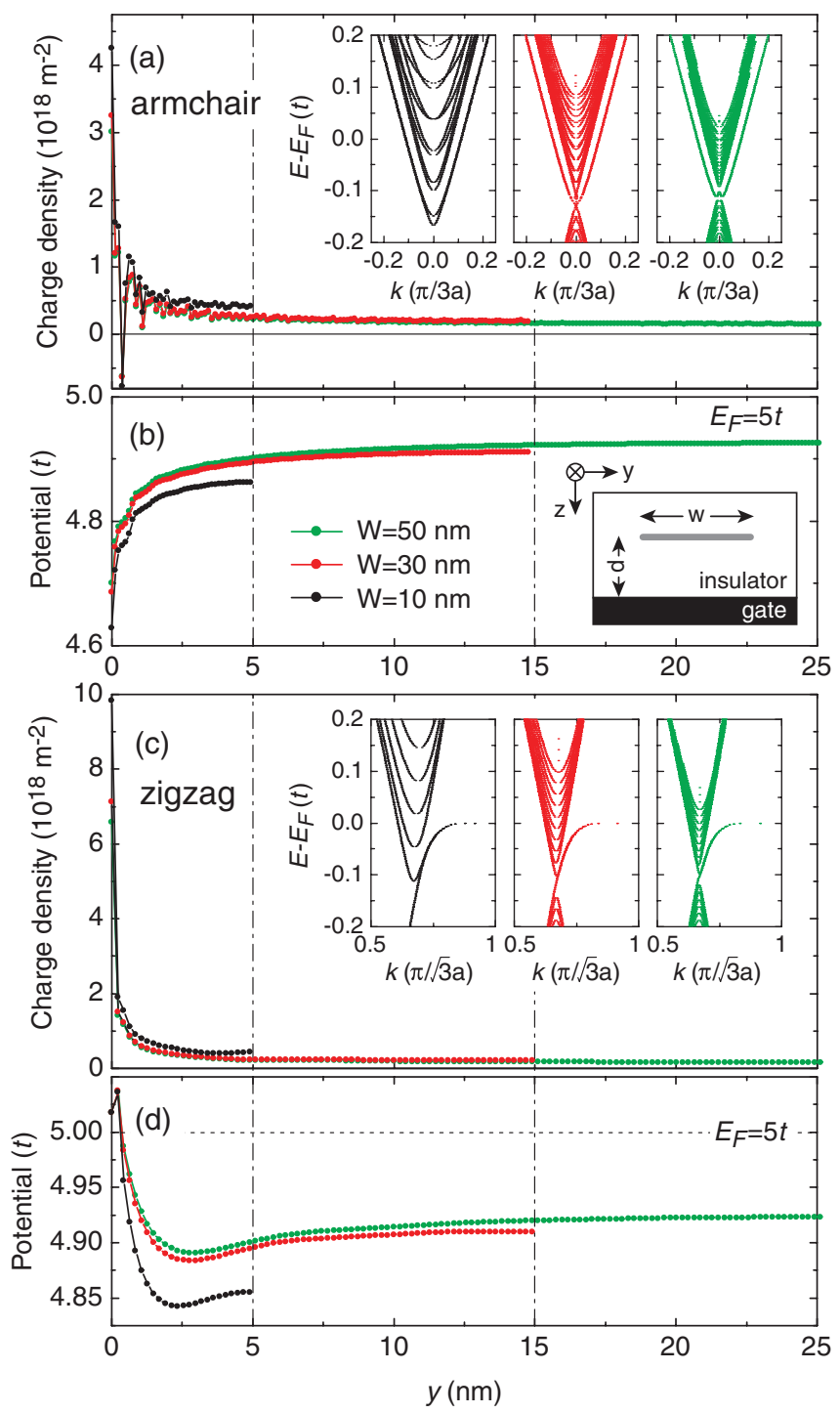

FIG. 1. (Color online) (a), (c) The self-consistent charge densities and (b), (d) potentials for the armchair and zigzag ribbons of widths $W=10,30$, and $50 \mathrm{~nm}$ calculated in the Hartree approximation at zero magnetic field. Only half of the ribbon is shown. The insets in (a) and (c) show the dispersion relation for nanoribbons of different widths $W=10,30$, and $50 \mathrm{~nm}$ from left to right for several of the lowest subbands. The inset in (b) shows the schematic geometry of the device; $d=30 \mathrm{~nm}$. $E_{F}=5 t, t=2.7 \mathrm{eV}$, and $a=0.142 \mathrm{~nm}$.

the $p$-orbital tight-binding Hubbard-type Hamiltonian in the mean-field approximation, $H=H^{\uparrow}+H^{\downarrow}$, which is shown to describe carbon electron systems in good agreement with the first-principles calculations, ${ }^{28,29}$

$$
\begin{aligned}
H^{\sigma}= & -\sum_{\mathbf{r}, \Delta} t_{\mathbf{r}, \mathbf{r}+\Delta} a_{\mathbf{r} \sigma}^{+} a_{\mathbf{r}+\Delta, \sigma} \\
& +\sum_{\mathbf{r}}\left[V_{Z}^{\sigma}+V_{H}(\mathbf{r})+V_{U}^{\sigma^{\prime}}(\mathbf{r})\right] a_{\mathbf{r} \sigma}^{+} a_{\mathbf{r} \sigma},
\end{aligned}
$$

where $\sigma$ and $\sigma^{\prime}$ correspond to two opposite spin states, $\uparrow$ and $\downarrow$; the summation runs over all sites $\mathbf{r}=(x, y)$ of the graphene lattice, and $\Delta$ includes the nearest neighbors only. The magnetic field is included in a standard way via Pierel's substitution, $t_{\mathbf{r}, \mathbf{r}+\Delta}=t_{0} \exp \left(i 2 \pi \phi_{\mathbf{r}, \mathbf{r}+\Delta} / \phi_{0}\right)$, where $\phi_{\mathbf{r}, \mathbf{r}+\Delta}=$
$\int_{\mathbf{r}}^{\mathbf{r}+\Delta} \mathbf{A} \cdot d \mathbf{l}$, with $\mathbf{A}$ being the vector potential, $\phi_{0}=h / e$ being the magnetic flux quantum, and $t_{0}=2.7 \mathrm{eV}$. [In our calculations we use the Landau gauge, $\mathbf{A}=(-B y, 0)$.] The first two terms in Eq. (1) correspond to the noninteracting part of the Hamiltonian, with the first term describing the kinetic energy of the electrons on a graphene lattice. The second term describes the Zeeman energy triggering the spin splitting in the magnetic field, $V_{Z}^{\sigma}= \pm \frac{1}{2} g \mu_{b} B$, where the $+(-)$ sign corresponds to the spin state $\uparrow(\downarrow) ; g=2$ is the bare $g$ factor of pristine graphene, and the Bohr magneton $\mu_{b}=e \hbar / 2 m_{e}$. The last two terms in Eq. (1) describe the electron interaction. The long-range Coulomb interaction between induced charges in the GNR is given by the standard Hartree term,

$$
V_{H}(\mathbf{r})=\frac{e^{2}}{4 \pi \varepsilon_{0} \varepsilon_{r}} \sum_{\mathbf{r}^{\prime} \neq \mathbf{r}} n_{\mathbf{r}^{\prime}}\left(\frac{1}{\left|\mathbf{r}-\mathbf{r}^{\prime}\right|}-\frac{1}{\sqrt{\left|\mathbf{r}-\mathbf{r}^{\prime}\right|^{2}+4 d^{2}}}\right),
$$

where $n_{\mathbf{r}}=n_{\mathbf{r} \uparrow}+n_{\mathbf{r} \downarrow}$ is the total electron density and the second term corresponds to a contribution from the mirror charges. The last term in Hamiltonian (1) corresponds to the Hubbard energy,

$$
V_{U}^{\sigma^{\prime}}(\mathbf{r})=U\left(n_{\mathbf{r}}^{\sigma^{\prime}}-\frac{1}{2}\right),
$$

and describes repulsion between electrons of opposite spins on the same site. The number of excess electrons at site $\mathbf{r}$ reads

$$
n_{\mathbf{r}}^{\sigma}=\int_{-\infty}^{\infty} \rho^{\sigma}(\mathbf{r}, E) f_{F D}\left(E, E_{F}\right) d E-n_{\text {ions }},
$$

where $\rho^{\sigma}(\mathbf{r}, E)=-\frac{1}{\pi} \operatorname{Im}\left[G^{\sigma}(\mathbf{r}, \mathbf{r}, E)\right]$ is the energy-dependent local density of states (LDOS) at zero temperature, $G^{\sigma}(\mathbf{r}, \mathbf{r}, E)$ is the Green's function in the real-space representation of an electron of spin $\sigma$ residing on site $\mathbf{r}, E_{F}=e V_{g}$ is the Fermi energy, which value is adjusted by the gate voltage, and $n_{\text {ions }}=1 / A_{0}=3.8 \times 10^{19} \mathrm{~m}^{-2}$ is the positive-charge background of ions $\left(A_{0}=\frac{3 \sqrt{3}}{4} a_{0}^{2}\right.$ is the area per one $\mathrm{C}$ atom and $a_{0}=0.142 \mathrm{~nm}$ is the C-C distance). Equations (1)-(4) are solved self-consistently using the Green's function technique in order to calculate the band structure, the charge density, and the potential distribution. ${ }^{30-32}$ For a given potential distribution we compute the conductance using the Landauer formula,

$$
\mathcal{G}^{\sigma}\left(E_{F}\right)=\frac{e^{2}}{h} \int T^{\sigma}(E)\left[-\frac{\partial f_{F D}\left(E-E_{F}\right)}{\partial E}\right] d E,
$$

where $T^{\sigma}(E)$ is the total transmission coefficient for electrons with spin $\sigma$ and $f_{F D}$ is the Fermi-Dirac distribution.

\section{RESULTS AND DISCUSSION}

\section{A. Potential profile and the charge accumulation near the edges in ribbons of different widths and edge terminations}

In the present study we aim to describe spin polarization in realistically wide GNRs. In current experiments ${ }^{33-36}$ the widths of nanoribbons are $W \sim 100 \mathrm{~nm}$, and the magnetic field reaches $B \lesssim 60 \mathrm{~T}$, corresponding to the ratio $W / l_{B} \approx 10$, with $l_{B}=\sqrt{\hbar / e B}$ being the magnetic length. At the same time, because of computational limitations, it is difficult to treat ribbons with widths exceeding $50 \mathrm{~nm}$. Therefore in our calculations we rescale the system using ribbons of smaller 
width $W \sim 30 \mathrm{~nm}$ subjected to higher fields (up to $B \sim 350 \mathrm{~T}$ ) in order to keep the ratio $W / l_{B} \approx 10$ in accordance with typical experiments. ${ }^{33-36}$ In very narrow ribbons the quantum confinement effects can dominate the ribbon's electronic properties. Thus, a concern might arise whether the obtained results remain valid for realistically wide ribbons. In the present section we investigate how a nanoribbon's electronic properties, such as the density distribution and the potential profile in the vicinity of the edge, change with the increase of the ribbon's width and find that the width of $W \approx 30 \mathrm{~nm}$ is already sufficient to capture all essential features of a wide ribbon or even a semi-infinite graphene sheet.

In our study we consider both types of edges, armchair and zigzag. We will demonstrate in subsequent sections that the main features in the spin polarization of the electron density and the enhancement of the $g$ factor are rather similar for both types of edges. There is, however, an important difference between them which can be traced to the presence of the zero-energy mode (ZEM) residing at the edge of the zigzag GNRs. ${ }^{37}$ In the present section we will demonstrate that the ZEM leads to different features in the potential and charge density profiles near the edges for the cases of armchair and zigzag ribbons.

Figure 1 shows the self-consistent charge density distributions and potential profiles for the armchair and zigzag nanoribbons of various widths $W=10,30,50 \mathrm{~nm}$. In all calculations the distance between the GNR and the gate is $d=30 \mathrm{~nm}$. The calculations are performed in the Hartree approximation for spinless electrons at zero field [i.e., $V_{U}^{\sigma}$ and $V_{Z}^{\sigma}$ are set to 0 in Hamiltonian (1)]. It is noteworthy that the electron-density distribution obtained from the electrostatics [i.e., due to the Hartree potential $V_{H}(\mathbf{r})$, Eq. (2)] is not altered significantly by the magnetic field. ${ }^{38}$ The charge densities and potentials stay qualitatively the same as the nanoribbon width increases and exhibit practically no difference for 30- and 50-nm-wide nanoribbons. We thus conclude that the transverse confinement does not change substantially for nanoribbons wider than $\sim 30 \mathrm{~nm}$, and the width $W=30 \mathrm{~nm}$ is sufficient to describe realistically wide ribbons or even an edge of a graphene sheet.

Let us now focus on a difference in the potential profiles and the electron-density distributions in the vicinity of a ribbon edge for armchair and zigzag ribbons. Both ribbons show strong electron accumulation near the edges, but this accumulation is stronger in the zigzag GNRs. The corresponding potential profiles for armchair and zigzag ribbons have different shapes near the edges. For the armchair ribbon, the potential has a triangular shape [see Fig. 1(b)]. This was predicted and explored previously. ${ }^{39,40}$ The triangular shape of the potential is related to the hard-wall confinement. It is noteworthy that a similar triangular shape of a potential is exhibited by cleaved-edge overgrown quantum wires where electrons also experience a hard-wall confinement. ${ }^{20,41}$

The potential profile for the case of zigzag ribbon exhibits somehow different features. As in the case of the armchair GNRs, it gradually decreases towards the boundaries to form a well in the vicinity of the edges. However, in close proximity to the edges, it raises up and crosses the Fermi energy [see Fig. 1(d)]. We relate this feature to the ZEM that traps charges. The zero-energy mode is manifested as a disperseless energy level pinned to $E_{F}$ in the ranges $k \in \frac{\pi}{\sqrt{3} a}\left[\frac{2}{3}, 1\right]$ and $k \in-\frac{\pi}{\sqrt{3} a}\left[\frac{2}{3}, 1\right]$. It is these trapped charges that raise the potential at the edges. They effectively repulse excess charges induced in the ribbon by the gate and prevent the triangular well from forming near the boundary. Therefore, the difference in the charge accumulation and potential profiles in the armchair and zigzag ribbons occurs due to the topological property of the zigzag edge termination, supporting the zero-energy mode. It is important to stress that this difference persists into the high-field regime and cannot be addressed by semiclassical approaches as in Ref. 39.

\section{B. LDOS, magnetoband structure, and formation of compressible strips for spinless electrons}

Spin polarization in conventional quantum wires is related to the formation of compressible strips $^{38}$ in the case of interacting spinless electrons. ${ }^{19-21}$ In this section we therefore outline the electronic and transport properties of armchair and zigzag nanoribbons in the Hartree approximation for spinless electrons [i.e., disregarding the Hubbard and Zeeman interactions, $V_{Z}=V_{U}=0$, in Hamiltonian (1)], focusing on the formation of the compressible strips.

Figures 2(a) and 2(b) show the local density of states (LDOS), $L(y)=-\int \rho(y, E) \frac{\partial f_{F D}\left(E-E_{F}\right)}{\partial E} d E$, and the total density of states (DOS), $D=\sum_{y} L(y)$, at the Fermi energy as a function of the magnetic field for the armchair and zigzag ribbons. (Note that LDOS is shown for one sublattice only.) It is noteworthy that the DOS can be accessible via magnetocapacitance or magnetoresistance measurements, similar to conventional semiconductor structures defined in 2DEG. ${ }^{42,43}$ The structure of the LDOS and DOS can be understood from an analysis of the magnetosubband structure. We outline below the main features of the subband structure for the armchair and zigzag ribbons, focusing on the differences and similarities between them as well as on formation of the compressible strips in the ribbons. (Note that the evolution of the band structure for the case of the armchair GNRs was discussed by Shylau et al. ${ }^{31}$ )

The left panels of Figs. 3(a)-3(c) show the band structure of armchair graphene nanoribbons for spinless Hartree electrons for three representative magnetic fields. Flat regions in the band diagrams correspond to the Landau levels in bulk graphene, and dispersiveness states close to the GNRs boundaries represent edge states corresponding to classical skipping orbits. Figure 3(a) shows the band diagram for a magnetic field $B=140$ T when the two lowest Landau levels, LL0 and LL1, are filled. The first Landau level, LL1, is pinned to the Fermi energy, thus forming a compressible strip in the center of the GNR. The strip is called compressible when the electron density can be easily redistributed in order to effectively screen the external potential. We define a compressible strip as a region where the dispersion lies within the energy window $\left|E-E_{F}\right| \lesssim 2 \pi k_{B} T$ (Refs. 18, 19,31, and 44) because in this energy window the states are partially filled, i.e., $0<f_{F D}<1$, and thus the electron density can be easily changed.

Because a graphene ribbon has abrupt edges, the selfconsistent potential forms the triangular wells near the edges, as discussed in the previous section. As a result the center 

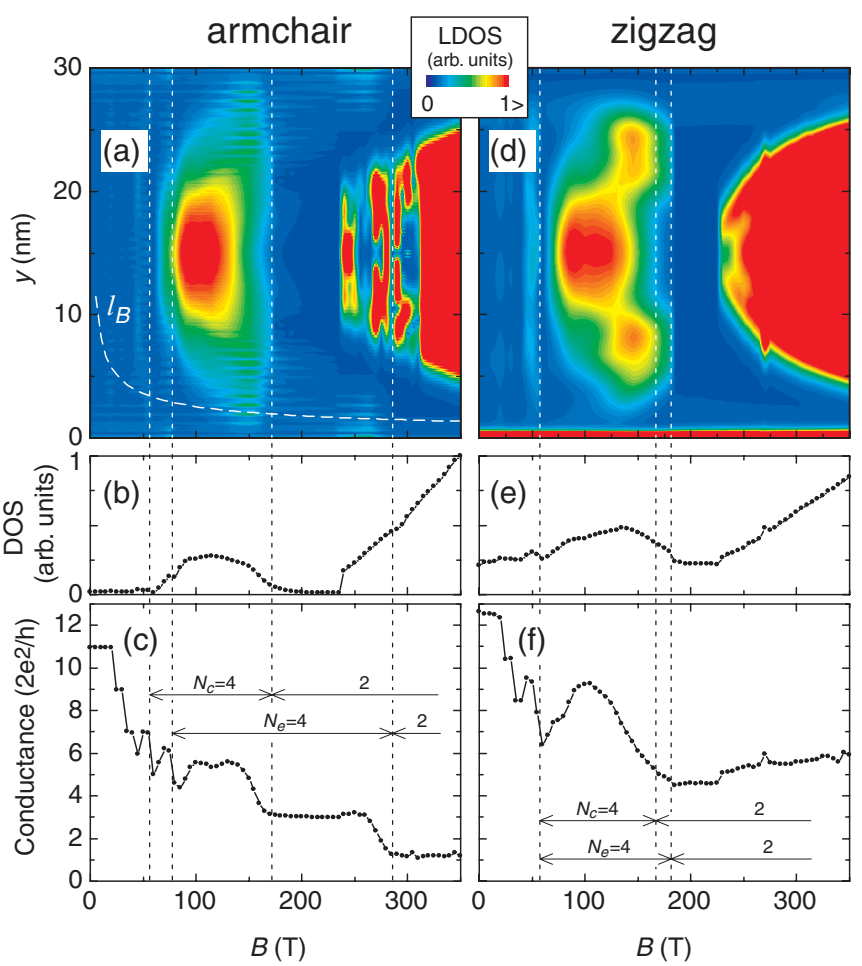

FIG. 2. (Color online) (a), (d) The LDOS and (b), (e) the DOS at the Fermi energy and (c), (f) the two-terminal conductance calculated in the Hartree approximation for the (left) armchair and (right) zigzag ribbons. The LDOS is shown for one graphene sublattice, say A. The LDOS for sublattice B is symmetric with respect to the ribbon's axis. The regions with higher LDOS point to a higher probability of finding an electron there and correspond to positions of the compressible strip. $N_{c}$ and $N_{e}$ in (c) and (f) mark the number of occupied electron subbands in the center and near the edge of the ribbon, respectively. The width of ribbons is $W=30 \mathrm{~nm}$, corresponding to 242 and 141 carbon atoms in the cross section of armchair and zigzag ribbons, respectively. $E_{F}=5 t$. Temperature $T=10 \mathrm{~K}$.

of the ribbon and its edges depopulate in the magnetic field differently. Namely, as the magnetic field increases, the subbands first depopulate in the center and then near the edges. For example, the second subband (i.e., LL1) is pinned to the Fermi level in the ribbon center in the interval $B \approx 60-170 \mathrm{~T}$. Within this interval it forms the compressible strip, which manifests as the enhanced LDOS and DOS in Figs. 2(a) and 2(b). However, the LL1 stays populated near the edges in a wider magnetic field range, $B \approx 80-290 \mathrm{~T}$. In the field interval $B \approx 170-230 \mathrm{~T}$ the LL1 is depopulated in the center [see Fig. 3(b)]. As a result the LDOS and DOS are practically zero [see Figs. 2(a) and 2(b)]. When the magnetic field increases to $B \approx 230 \mathrm{~T}$, the lowest Landau level, LL0, is pushed up in energy and gets pinned to the Fermi energy. This again leads to the formation of the compressible strip in the middle of the wire [see Fig. 3(b)] and to the enhancement of the LDOS and DOS at the Fermi energy, as seen in Figs. 2(a) and 2(b). At this $B$, two different LLs are at $E_{F}$ and contribute to electron transport: LLO in the center and LL1 near the edges.

The graphene ribbons with the zigzag edge termination exhibit features of the magnetosubband structure similar to those for the armchair-terminated ribbons. This is illustrated in Figs. 3(d)-3(f), which show the band structure of the zigzag GNRs for three representative magnetic fields, $B=90,220$, and $300 \mathrm{~T}$. As for the armchair GNRs, these fields correspond respectively to the cases when the LL1 is pinned to $E_{F}$ in the middle of the GNR [Fig. 3(d)], LL1 is depopulated in the middle of the GNR [Fig. 3(e)], and LL0 is pinned to $E_{F}$ in the middle of the GNR [Fig. 3(f)]. However, there are several striking differences between the zigzag and armchair ribbons manifested in their LDOS, DOS, and the subband structure. First, strong electron accumulation and formation of the compressible strip takes place near the ribbon's boundaries over the whole range of magnetic fields studied; see the enhanced LDOS at $y \sim 0$ in Fig. 2(d). Because of this the total DOS in the zigzag GNR never drops to zero over the whole range of magnetic fields [see Fig. 2(e)]. [Note that Fig. 2(d) shows the LDOS for sublattice A, which is enhanced at one edge of the ribbon. Sublattice B has the enhanced LDOS near the opposite edge of the ribbon.]

Inspection of the magnetoband structure reveals that these features are caused by the ZEM in the zigzag GNR discussed in the previous section. This mode [marked ZEM in Figs. 3(d)3(f)] always stays pinned to the Fermi energy because of the high density of states for electrons that this mode accommodates. It is important to stress that the pinning of this mode to $E=E_{F}$ is the result of the electron-electron interaction, and the pinning effect is apparently absent in the one-electron description where this mode is always situated at $E=0 .{ }^{37}$

Figures 2(c) and 2(f) show the evolution of the two-terminal conductance as the magnetic field increases for the armchair and zigzag ribbons. In contrast to the conventional semiconductor quantum wires and quantum point contacts exhibiting a steplike conductance, ${ }^{45}$ the conductance of GNRs reveals a nonmonotonic decrease with bumps coexisting with quantized plateau regions of multiples of $\frac{2 e^{2}}{h}$. Note that $\mathcal{G}$ changes by two conductance quanta between plateaus due to valley degeneracy of graphene. The origin of the bumps in the conductance was discussed by Shylau et al. ${ }^{31}$ and was related to the interaction-induced modifications of the band structure leading to the formation of compressible strips in the middle of GNRs.

\section{Spin splitting and the enhancement of the $g$ factor}

Let us now turn to the case of electrons with spin and analyze how the Hubbard and the Zeeman interactions modify the magnetosubband structure of graphene nanoribbons, leading to the spin polarization and enhancement of the $g$ factor. For the Hubbard constant we choose $U=t$, which corresponds to the estimation of Refs. 29 and 46 . Note that a recent work ${ }^{47}$ predicts a somehow larger value, $U \approx 3 t$. While the utilization of a larger value of $U$ leads to some quantitative differences with the results presented below, they remain qualitatively the same, and the conclusions are not affected. We also stress that while the discussion below is focused on the case of high magnetic field when the two lowest Landau levels, LL1 and LL0, are occupied, the conclusion remains valid for lower fields as well.

Let us start with the case of armchair ribbons. Figures 4(a)-4(c) show the local spin polarization of the charge density, $\zeta(y)=\frac{n^{\uparrow}(y)-n^{\downarrow}(y)}{n^{\uparrow}(y)+n^{\downarrow}(y)}$, spin-resolved densities, and the 

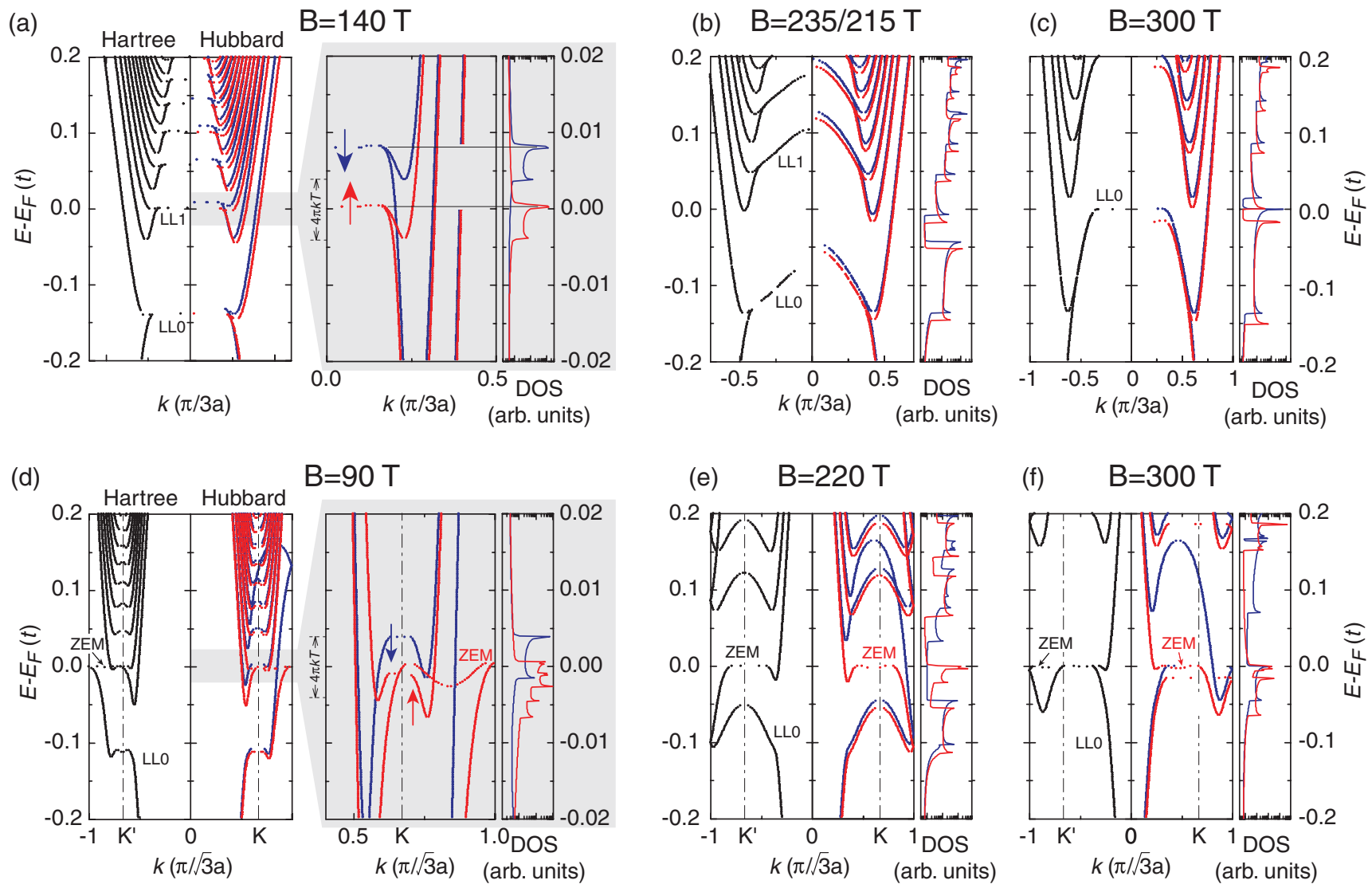

FIG. 3. (Color online) The dispersion relation for (a)-(c) the armchair and (d)-(f) zigzag ribbons. The left and right panels in each plot correspond, respectively, to the Hartree and Hubbard approximations. Because of the symmetry, only half of the band diagram is shown. The red and blue dotted lines show, respectively, the spin-up and spin-down components. Vertical dot-dashed lines in (d)-(f) mark the $K$ and $K^{\prime}$ points of the first Brillouin zone of graphene in the zigzag ribbon. In the armchair ribbon, the $K$ and $K^{\prime}$ points coincide at $k=0$. In (d)-(f), ZEM marks the zero-energy mode that stays pinned to the Fermi energy.

total spin polarization, $P=\frac{n^{\uparrow}-n^{\downarrow}}{n^{\uparrow}+n^{\downarrow}}\left[n^{\sigma}=\sum_{y} n^{\sigma}(y)\right]$, as a function of magnetic field. The features in $\zeta(y)$ show a striking similarity with the features of the LDOS, and the behavior of $P$ follows that of the DOS calculated in the Hartree approximation [cf. Figs. 2(a) and 2(b)]. This similarity is not coincidental. The regions with the enhanced LDOS correspond to compressible strips, and it is the compressible strips where the spin splitting of subbands takes place. Indeed, in the compressible region the subbands are only partially filled because $f_{F D}<1$ there, and therefore the population of the spin-up and spin-down subbands can be easily changed. This population difference triggered by Zeeman splitting is enhanced by the Hubbard interaction, leading to different effective potentials for spin-up and spin-down electrons and eventually to the subband spin splitting.

For a more detailed analysis let us follow an evolution of the band structure in Figs. 3(a)-3(c). The right panel of Fig. 3(a) shows a spin-resolved magnetoband structure corresponding to the case when LL1 forms a compressible strip in the middle of the ribbon for the case of spinless electrons (cf. the left panel). The Hubbard interaction pushes up the spin-down subbands above the window $\left|E-E_{F}\right| \approx 2 k_{B} T$ such that it becomes depopulated, and the compressible strip in the middle is occupied by spin-up electrons only. As a result, the DOS at $E_{F}$ of the spin-up electrons is enhanced, while that of the spin-down electrons is zero [see Fig. 4(d)]. All these lead to the difference in the electron densities $n^{\uparrow}$ and $n^{\downarrow}$ and the spin polarization in the ribbon, as shown in Figs. 4(b) and 4(c). When the magnetic field increases such that the LL1 is pushed above $E_{F}$, the compressible strip in the middle disappears, the DOS at $E_{F}$ for both spin species becomes equal to zero, and the spin polarization vanishes. This is illustrated in the band diagram shown in Fig. 3(b), corresponding to the case when $E_{F}$ is situated between LLO and LL1. With a further increase of the magnetic field the LLO is pushed up to $E_{F}$. As this subband is pushed from below, in this case, it is the higher-energy spin-down state that gets pinned to $E_{F}$, forming compressible strips, whereas the spin-up subband in the middle of the ribbon remains below $\left|E-E_{F}\right| \approx 2 k_{B} T$. As a result, for this case the DOS at $E_{F}$ for the spin-down electrons is larger than that for the spin-up electrons [Fig. 4(d)]. Note that despite this, $n^{\uparrow}>n^{\downarrow}$ [see Fig. 4(b)] because the spin-up subband is fully occupied, whereas the spin-down subband is occupied only partially.

Figure 4(e) shows the effective $g^{*}$ factor for the armchair ribbon, defined according to

$$
g^{*}=\frac{\left\langle V^{\uparrow}-V^{\downarrow}\right\rangle}{\mu_{B} B},
$$

where the averaging is done over all carbon atoms. Because the electron density is related to the potential, the features in 

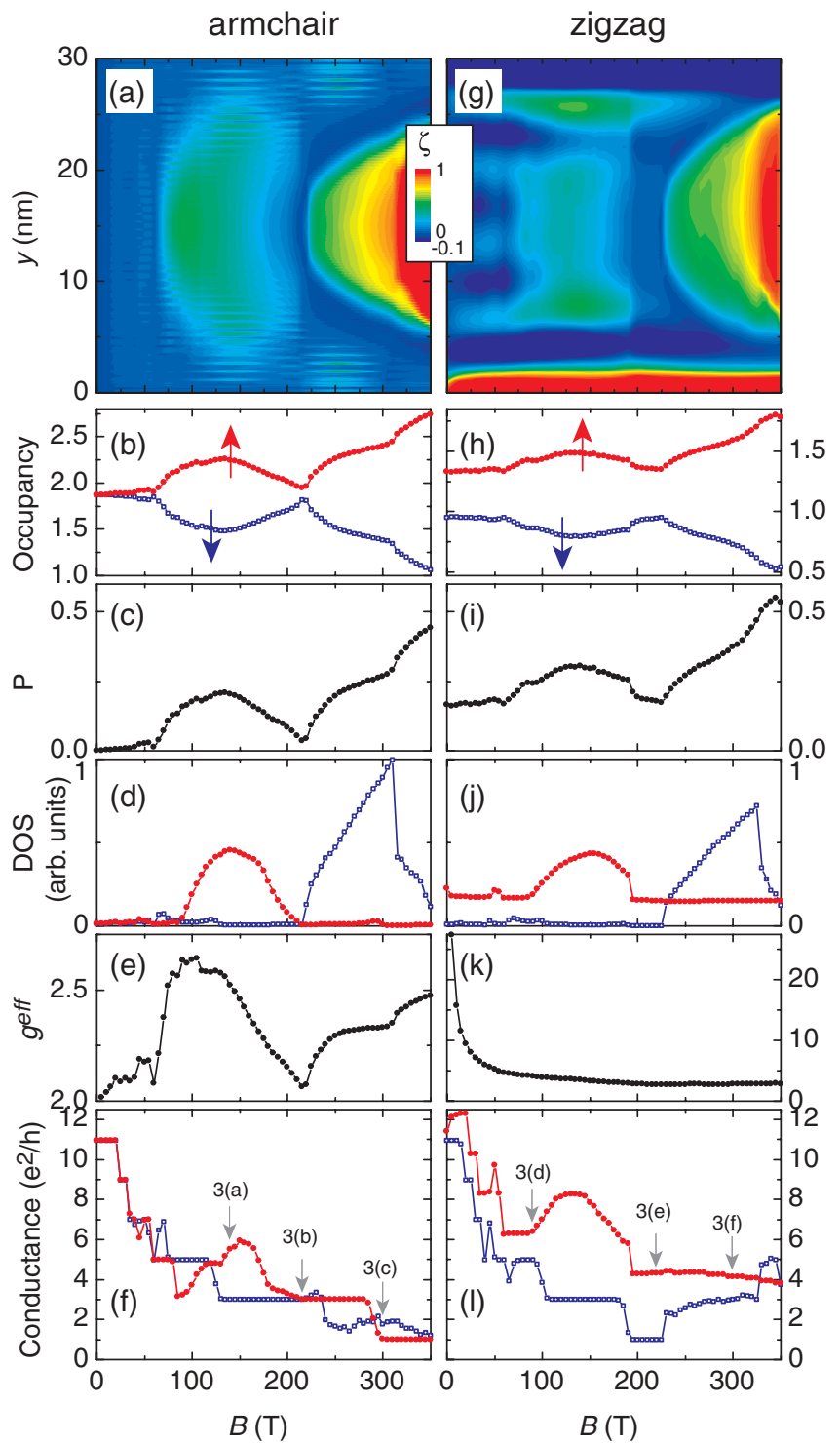

FIG. 4. (Color online) (a), (g) The local spin polarization of the charge density for sublattice A. The polarization for sublattice B (not shown here) is symmetric with respect to the ribbon's axis. (b), (h) The unit cell occupancy, (c), (i) the spin polarization $P,(\mathrm{~d}),(\mathrm{j})$ the DOS, (e), (k) the effective $g$ factor, and (f), (l) the conductance as a function of magnetic field calculated in the Hubbard approximation for the (left) armchair and (right) zigzag ribbons. The red lines with solid dots show the spin-up component, and the blue lines with open squares show the spin-down component. Arrows in (f) and (l) mark magnetic fields for the band structures shown in Fig. 3.

the $g^{*}$ factor resemble those of the polarization $P$, showing behavior reflecting successive population and depopulation of the spin-up and spin-down subbands.

In high magnetic field, $B \gtrsim 50 \mathrm{~T}$ (corresponding to population of LL1 and LL0), the effective $g$ factor varies in the range $2.1 \lesssim g^{*} \leqslant 2.7$, with an average value of $\left\langle g^{*}\right\rangle \approx 2.25$, which represents a rather modest enhancement in comparison to the case of noninteracting electrons in pristine graphene with $g=2$. Note that in the bulk graphene the effective $g^{*}$ factor was reported to be $g^{*} \approx 2.7 .^{10}$
It is worth mentioning that the main features of the spin polarization and the subband evolution in the magnetic field resemble those in the cleaved-edge overgrown quantum wires $(\mathrm{CEOQW}){ }^{20}$ This is because in both cases the potential corresponds to the hard-wall confinement. The difference is that in CEOQW, as well as in conventional GaAs split-gate wires ${ }^{18}$ the polarization and thus the effective $g^{*}$ factor are enhanced by a factor of $\sim 10$ compared to the Zeeman splitting, whereas in the armchair ribbons this enhancement it is just $\sim 0.22$. This can be explained by the fact that the bare $g$ factor in armchair GNRs is much larger than that in GaAs $\left(g_{\text {GaAs }} / g_{\text {graphene }} \approx 0.022\right)$, such that the Zeeman interaction in graphene remains dominant compared to the exchange one.

One more important difference of the graphene ribbons from the conventional GaAs quantum wires is in the character of the spin-polarized edge states in the vicinity of the boundaries. In the conventional quantum wires the edge states of opposite spins are spatially separated. ${ }^{19}$ This is ultimately related to the formation of the compressible strips near the boundaries of the split-gate wire because of the soft confinement due to the gates. ${ }^{19}$ In GNR due to the hard-wall confinement the compressible strips do not form near the boundaries, and hence the spatial separation of the edge states of opposite spins does not occur. It is worth mentioning that in this respect the GNRs are also similar to CEOQW. ${ }^{20}$

Let us now turn to the case of zigzag nanoribbons. The main features of the spin polarization and the subband evolution at high fields are rather similar to the armchair ones. There are, however, important differences, especially at low fields, related to the presence of the zero-energy mode residing at the zigzag edges. In contrast to other modes exhibiting successive population and depopulation of the Landau levels in the middle of the ribbon, this mode always stays pinned to $E_{F}$, thus forming a compressible strip with the enhanced density of states at the edges for all magnetic fields. The Hubbard interaction leads to a complete spin polarization such that electrons in this mode are always in the spin-up state. This is seen in the spatially resolved polarization shown in Fig. 4(g). Because of this the total spin-up density is significantly larger than the spin-down one for all magnetic fields, and the DOS for spin-up electrons never drops to zero. This is in contrast to the case of armchair GNRs, where electron densities for opposite spin species can be equal when the Fermi energy lies between two consecutive Landau levels and where both DOS for spin ups and spin downs can drop to zero [cf. Fig. 4(d) with Fig. 4(j)].

Figure 4(k) shows the effective $g$ factor for the case of zigzag GNRs. In contrast to the case of armchair GNRs, where the effective $g$ factor at low fields $(B \lesssim 50 \mathrm{~T}$ ) is not much different from the bare $g$ factor of pristine graphene, the effective $g$ factor in zigzag GNRs is enhanced up to values of $g^{*} \approx 30$. At higher fields it decreases to values of $g^{*} \approx 3$, comparable to those in armchair GNRs. This striking feature is another manifestation of the existence of the spin-polarized ZEM in the zigzag GNR. Indeed, as seen in Fig. 2(d), the spin polarization of the ZEM is rather constant over the entire range of the magnetic fields studied. As a result, at low fields when the polarization of other states is rather low, the potential difference $\left\langle V^{\uparrow}-V^{\downarrow}\right\rangle$ determining $g^{*}$ according to Eq. (6), is primarily 
due to the contribution from the ZEM and thus weakly depends on $B$. At the same time, because the field $B$ enters the denominator of Eq. (6), the effective $g$ factor gets strongly enhanced when $B$ is low. As $B$ increases, other states become spin polarized due to the Zeeman and Hubbard terms, and the contribution of the ZEM in the overall spin polarization decreases. As a result, $\left\langle V^{\uparrow}-V^{\downarrow}\right\rangle$ becomes proportional to $B$, which leads to stabilization of $g^{*}$ around a constant value $\left(g^{*} \approx 3\right)$.

Figures 4(f) and 4(1) show the evolution of the twoterminal conductance as the magnetic field increases for the armchair and zigzag ribbons. The conductance is apparently spin polarized with $\mathcal{G}^{\uparrow} \neq \mathcal{G}^{\downarrow}$. This reflects the fact that at a given magnetic field the numbers of propagating states at $E_{F}$ accommodating spin-up and spin-down electrons are different. As in the case of spinless electrons, the spin-resolved conductance also exhibits a bumplike structure whose origin is the same as for the case of spinless electrons. For zigzag GNRs the spin-up conductance is larger than the spin-down one for most fields because of the presence of a spin-polarized ZEM that always contributes to the electrical conduction. While being pinned to $E_{F}$, it is only partly populated and therefore gives rise to the conductance that deviates from multiples of the conductance quanta. As a result, the conductance quantization is less pronounced in zigzag GNRs compared to armchair ones. Finally, we stress that Figs. 4(f) and 4(1) show the conductances of ideal GNRs without defects. The defect scattering will modify the GNR conductance, especially for the low-velocity modes flowing in the middle of the ribbons. At the same time, the edge states corresponding to the classical skipping orbits are robust against the impurity scattering. ${ }^{31}$

Finally, we note that we also performed similar computations of the spin polarization for GNRs using the density functional theory with the exchange functional proposed by Polini et al. ${ }^{48}$ including the spin degree of freedom as prescribed in Ref. 49. Practically no spin polarization was observed that we attribute to the positive sign of the exchange energy in Ref. 48. More systematic studies of the spin polarization in GNRs using different approaches (such as the spin DFT, Hartree-Fock, etc.) would be very interesting. Note that spin polarization and the enhancement of the $g$ factor in bulk graphene in the presence of impurities was recently studied by Volkov et al ${ }^{50}$ within the Thomas-Fermi approximation.

\section{CONCLUSIONS}

We provided a systematic quantitative description of the spin polarization, the subband structure, and the density and potential profiles in the armchair and zigzag graphene nanoribbons in a perpendicular magnetic field. In our study we addressed realistically wide nanoribbons, and our conclusion concerning the density and potential distributions near the edge can also be applied for the case of a semi-infinite graphene sheet. Our calculations are based on the self-consistent Green's function technique where electron interaction and spin effects are included by the Hartree and Hubbard potentials.

We first focused on the case of spinless electrons and found that the potential profile and the density distribution are different near the edges of the armchair and zigzag ribbons. For the armchair termination, the potential at the edge has a triangular shape, whereas for the zigzag ribbons it exhibits a well-type character. Both terminations show strong electron accumulation near the edges, but this accumulation is stronger at the zigzag edge. This difference is attributed to a topological property of the zigzag edge termination supporting the zero-energy mode.

Because the spin polarization in nanoribbons and conventional quantum wires is ultimately related to the formation of the compressible strips for the case of spinless electrons, we studied the LDOS, DOS, and magnetosubband structure for the armchair and zigzag ribbons, focusing on the differences and similarities between them as well as on the formation of the compressible strips in the ribbons. For both types of nanoribbons we found that a compressible strip with the enhanced DOS forms in the middle of the ribbon, in accordance with the successive population and depopulation of the Landau levels. For the case of zigzag edge termination we found a strong electron accumulation and formation of a compressible strip near the edges over the whole range of magnetic fields. This is caused by the presence of the zero-energy mode that always stays pinned to the Fermi energy because of the high DOS that this mode accommodates.

Accounting for the Zeeman interaction and describing the spin effects via the Hubbard potential, we discussed how the spin-resolved subband structure evolves when an applied magnetic field varies. We found that the local spin polarization of the electron density and the total spin polarization exhibit a behavior similar to that of the LDOS and DOS for spinless electrons. This similarity is not coincidental and reflects the fact that the regions with the enhanced DOS correspond to compressible strips where the spin splitting of subbands takes place. We found that for the armchair ribbons in high magnetic field the effective $g$ factor varies in the range $2.1 \lesssim g^{*} \leqslant 2.7$, with an average value of $\left\langle g^{*}\right\rangle \approx 2.25$. For the zigzag nanoribbons we found that the zero-energy mode remains pinned to $E_{F}$ and becomes fully spin polarized for all magnetic fields, which, in turn, leads to a strong spin polarization of the electron density near the zigzag edge. Due to the contribution of the fully spin-polarized zero-energy mode the effective $g^{*}$ factor in zigzag GNRs is strongly enhanced at low field, reaching values up to $g^{*} \approx 30$, and at higher fields it decreases to values of $g^{*} \approx 3$, comparable to those in armchair GNRs.

It is worth mentioning that the main features of the spin polarization and the subband evolution in the magnetic field resemble those in the cleaved-edge overgrown quantum wires. This is because in both cases the potential corresponds to the hard-wall confinement.

Finally, we stress the importance of accounting for the global electrostatics in the system at hand to accurately describe the spin polarization in GNRs. (In the present study it is done by accounting for the long-range Coulomb interaction by means of the self-consistent Hartree potential.) This is because the global electrostatic is responsible for the formation of the compressible strips, and it is the compressible strips where the spin splitting of subbands takes place.

\section{ACKNOWLEDGMENT}

The authors acknowledge a collaborative grant from the Swedish Institute. 
*sihnatse@sfu.ca

†igor.zozoulenko@liu.se

${ }^{1}$ A. H. Castro Neto, F. Guinea, N. M. R. Peres, K. S. Novoselov, and A. K. Geim, Rev. Mod. Phys. 81, 109 (2009).

${ }^{2}$ V. P. Gusynin and S. G. Sharapov, Phys. Rev. Lett. 95, 146801 (2005).

${ }^{3}$ K. S. Novoselov, A. K. Geim, S. V. Morozov, D. Jiang, M. I. Katsnelson, I. V. Grigorieva, S. V. Dubonos, and A. A. Firsov, Nature (London) 438, 197 (2005).

${ }^{4}$ Y. Zhang, Y.-W. Tan, H. L. Stormer, and P. Kim, Nature (London) 438, 201 (2005).

${ }^{5}$ Y. Zhang, Z. Jiang, J. P. Small, M. S. Purewal, Y.-W. Tan, M. Fazlollahi, J. D. Chudow, J. A. Jaszczak, H. L. Stormer, and P. Kim, Phys. Rev. Lett. 96, 136806 (2006).

${ }^{6}$ Z. Jiang, Y. Zhang, H. L. Stormer, and P. Kim, Phys. Rev. Lett. 99, 106802 (2007).

${ }^{7}$ J. G. Checkelsky, L. Li, and N. P. Ong, Phys. Rev. Lett. 100, 206801 (2008).

${ }^{8}$ L. Zhang, J. Camacho, H. Cao, Y. P. Chen, M. Khodas, D. E. Kharzeev, A. M. Tsvelik, T. Valla, and I. A. Zaliznyak, Phys. Rev. B 80, 241412(R) (2009).

${ }^{9}$ Y. Zhao, P. Cadden-Zimansky, F. Ghahari, and P. Kim, Phys. Rev. Lett. 108, 106804 (2012).

${ }^{10}$ E. V. Kurganova, H. J. van Elferen, A. McCollam, L. A. Ponomarenko, K. S. Novoselov, A. Veligura, B. J. van Wees, J. C. Maan, and U. Zeitler, Phys. Rev. B 84, 121407(R) (2011).

${ }^{11}$ M. B. Lundeberg and J. A. Folk, Nat. Phys. 5, 894 (2009).

${ }^{12}$ J. Güttinger, T. Frey, C. Stampfer, T. Ihn, and K. Ensslin, Phys. Rev. Lett. 105, 116801 (2010).

${ }^{13}$ J. M. Kinaret and P. A. Lee, Phys. Rev. B 42, 11768 (1990).

${ }^{14}$ J. Dempsey, B. Y. Gelfand, and B. I. Halperin, Phys. Rev. Lett. 70, 3639 (1993).

${ }^{15}$ Y. Tokura and S. Tarucha, Phys. Rev. B 50, 10981 (1994).

${ }^{16}$ Z. Zhang and P. Vasilopoulos, Phys. Rev. B 66, 205322 (2002).

${ }^{17}$ T. H. Stoof and G. E. W. Bauer, Phys. Rev. B 52, 12143 (1995).

${ }^{18}$ S. Ihnatsenka and I. V. Zozoulenko, Phys. Rev. B 73, 075331 (2006).

${ }^{19}$ S. Ihnatsenka and I. V. Zozoulenko, Phys. Rev. B 73, 155314 (2006).

${ }^{20}$ S. Ihnatsenka and I. V. Zozoulenko, Phys. Rev. B 74, 075320 (2006).

${ }^{21}$ S. Ihnatsenka and I. V. Zozoulenko, Phys. Rev. B 78, 035340 (2008).

${ }^{22}$ I. Žutić, J. Fabian, and S. Das Sarma, Rev. Mod. Phys. 76, 323 (2004).

${ }^{23}$ I. V. Zozoulenko and M. Evaldsson, Appl. Phys. Lett. 85, 3136 (2004).

${ }^{24}$ B. Karmakar, D. Venturelli, L. Chirolli, F. Taddei, V. Giovannetti, R. Fazio, S. Roddaro, G. Biasiol, L. Sorba, V. Pellegrini, and F. Beltram, Phys. Rev. Lett. 107, 236804 (2011).

${ }^{25}$ F. E. Camino, W. Zhou, and V. J. Goldman, Phys. Rev. B 72, 155313 (2005).
${ }^{26}$ S. Ihnatsenka, I. V. Zozoulenko, and G. Kirczenow, Phys. Rev. B 80, 115303 (2009).

${ }^{27}$ N. Paradiso, S. Heun, S. Roddaro, D. Venturelli, F. Taddei, V. Giovannetti, R. Fazio, G. Biasiol, L. Sorba, and F. Beltram, Phys. Rev. B 83, 155305 (2011).

${ }^{28}$ J. Fernández-Rossier and J. J. Palacios, Phys. Rev. Lett. 99, 177204 (2007).

${ }^{29}$ O. V. Yazyev, Phys. Rev. Lett. 101, 037203 (2008).

${ }^{30}$ H. Xu, T. Heinzel, M. Evaldsson, and I. V. Zozoulenko, Phys. Rev. B 77, 245401 (2008).

${ }^{31}$ A. A. Shylau, I. V. Zozoulenko, H. Xu, and T. Heinzel, Phys. Rev. B 82, 121410(R) (2010).

${ }^{32}$ A. A. Shylau and I. V. Zozoulenko, Phys. Rev. B 84, 075407 (2011).

${ }^{33}$ F. Molitor, A. Jacobsen, C. Stampfer, J. Güttinger, T. Ihn, and K. Ensslin, Phys. Rev. B 79, 075426 (2009).

${ }^{34}$ J. B. Oostinga, B. Sacepe, M. F. Craciun, and A. F. Morpurgo, Phys. Rev. B 81, 193408 (2010).

${ }^{35}$ J.-M. Poumirol, A. Cresti, S. Roche, W. Escoffier, M. Goiran, X. Wang, X. Li, H. Dai, and B. Raquet, Phys. Rev. B 82, 041413(R) (2010).

${ }^{36}$ R. Ribeiro, J.-M. Poumirol, A. Cresti, W. Escoffier, M. Goiran, J.-M. Broto, S. Roche, and B. Raquet, Phys. Rev. Lett. 107, 086601 (2011).

${ }^{37}$ K. Wakabayashi, M. Fujita, H. Ajiki, and M. Sigrist, Phys. Rev. B 59, 8271 (1999).

${ }^{38}$ D. B. Chklovskii, B. I. Shklovskii, and L. I. Glazman, Phys. Rev. B 46, 4026 (1992); D. B. Chklovskii, K. A. Matveev, and B. I. Shklovskii, ibid. 47, 12605 (1993)

${ }^{39}$ P. G. Silvestrov and K. B. Efetov, Phys. Rev. B 77, 155436 (2008).

${ }^{40}$ A. A. Shylau, J. W. Klos, and I. V. Zozoulenko, Phys. Rev. B 80, 205402 (2009).

${ }^{41}$ M. Huber, M. Grayson, M. Rother, W. Biberacher, W. Wegscheider, and G. Abstreiter, Phys. Rev. Lett. 94, 016805 (2005).

${ }^{42}$ D. Weiss, C. Zhang, R. R. Gerhardts, K. v. Klitzing, and G. Weimann, Phys. Rev. B 39, 13020 (1989).

${ }^{43}$ K.-F. Berggren, G. Roos, and H. van Houten, Phys. Rev. B 37, 10118 (1988).

${ }^{44}$ T. Suzuki and T. Ando, Phys. B 249-251, 415 (1998).

${ }^{45}$ C. W. J. Beenakker and H. van Houten, Solid State Phys. 44, 1 (1991).

${ }^{46}$ J. Jung and A. H. MacDonald, Phys. Rev. B 80, 235417 (2009).

${ }^{47}$ T. O. Wehling, E. Şaşioğlu, C. Friedrich, A. I. Lichtenstein, M. I. Katsnelson, and S. Blügel, Phys. Rev. Lett. 106, 236805 (2011).

${ }^{48}$ M. Polini, A. Tomadin, R. Asgari, and A. H. MacDonald, Phys. Rev. B 78, 115426 (2008).

${ }^{49}$ G. F. Giuliani and G. Vignale, Quantum Theory of the Electron Liquid (Cambridge University Press, Cambridge, 2005).

${ }^{50}$ A. Volkov, A. A. Shylau, and I. V. Zozoulenko, arXiv:1208.0522. 